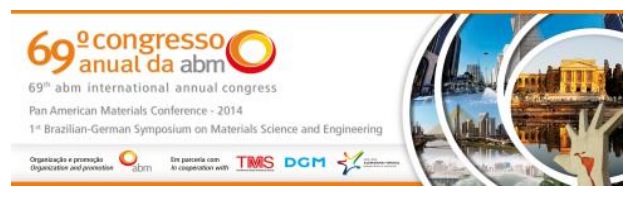

Tema: Corrosão

\title{
ANÁLISE COMPARATIVA DO COMPORTAMENTO CORROSIVO NA SOLDA DISSIMILAR DE LIGAS EXPERIMENTAIS COM ALTO TEOR DE MOLIBDÊNIO: ESTUDO DA CORROSÃO EM LIGAS EXPERIMETAIS*
}

\author{
Luana de Sousa Santos \\ Francisco Henrique Ximenes da $\mathrm{Cruz}^{2}$ \\ Víctor Moita Pinheiro ${ }^{3}$ \\ Marcelo Monteiro Valente Parente 4 \\ Rodrigo Freitas Guimães ${ }^{4}$
}

\section{Resumo}

O setor Petróleo e Gás Natural vem tendo enormes gastos com as paradas de manutenção. Estes gastos estão relacionados à degradação sofrida por seus equipamentos de refino devido à corrosão. Uma possível solução seria a utilização de novas ligas de aço inoxidável ferrítico com elevados percentuais de molibdênio, o que teoricamente favoreceria um aumento da resistência à corrosão. No entanto, pelo fato dessas ligas serem aplicadas na condição como soldada mudanças microestruturais significativas podem ser geradas, além de considerar o fato da solda das ligas em estudo ser realizada com material dissimilar, e a diferença existente entre o metal de adição e a região afetada pelo calor (ZAC) pode propiciar ao surgimento do fenômeno eletroquímico conhecido como pilha galvânica. Portanto, o objetivo deste trabalho foi analisar o comportamento corrosivo na solda dissimilar de duas novas ligas de aço inoxidável ferrítico com diferentes teores de cromo e um elevado teor de molibdênio, ambas com adicão de superliqa de níquel, verificando a influência da pilha. $O$ resultado da soldaqem foi avaliado através de exames metaloqráficos e ensaios eletroquímicos. As microqrafias do metal de adição utilizado na soldaqem das liqas mostraram que o mesmo possui uma microestrutura dendrítica e os qráficos obtidos dos ensaios de Polarização Potenciodinâmica Cíclica não reproduziram para as liqas soldadas, o que foi melhor analisado observando os resultados dos ensaios de forma separada, para as reqiões que constituem a solda das liqas, o metal de adição e ZAC. Sendo possível verificar a existência de uma maior resistência a corrosão no metal de adição.

Palavras-chave: Corrosão naftênica; Ligas experimentais; Molibdênio.

\section{COMPARATIVE ANALYSIS OF CORROSION BEHAVIOR IN WELDING ALLOYS DISSIMILAR EXPERIMENTAL HIGH LEVELS OF MOLYBDENUM: STUDY ON CORROSION OF ALLOY EXPERIMENTAL}

\section{Abstract}

The Oil and Natural Gas sector has had huge expenses on maintenance shutdowns. These expenses are related to the degradation suffered by their refining equipment due to corrosion. One possible solution would be to use new alloy ferritic stainless steel with high percentages of molybdenum, which theoretically would favor an increase in corrosion resistance. However, because these alloys are applied in welded condition as significant microstructural changes can be generated in addition to considering the fact solder alloys under study be performed with dissimilar material, and the difference between the weld metal and heat affected zone (HAZ) can facilitate the emergence of the phenomenon known as galvanic electrochemical cell. Therefore, the aim of this study was to analyze the corrosion behavior in dissimilar welding of two new ferritic stainless steel alloys with different chromium contents and a high molybdenum content, both with addition of nickel superalloy, and the influence of the pile. The result of the welding was evaluated by metallographic tests and electrochemical tests. The micrographs of addition used in the welding of metal alloys showed that it has a dendritic microstructure and the graphs obtained from the cyclic potentiodynamic polarization tests did not reproduce welded to the alloy which was analyzed by observing the best results of the tests separately, for regions that constitute the solder alloys, the weld metal and HAZ. Possible to verify the existence of a higher corrosion resistance in the weld metal.

Keywords: Naphthenic corrosion; Experimental alloys; Molybdenum.

1 Graduanda, Tecnologia em Manutenção Industrial, Instituto Federal de Educação, Ciência e Tecnologia do Ceará, Maracanaú, CE, Brasil.

2 Graduando, Engenharia Ambiental, Instituto Federal de Educação, Ciência e Tecnologia do Ceará, Maracanaú, CE, Brasil.

3 Físico, Universidade Federal do Ceará, Fortaleza, CE, Brasil.

4 Engenheiro Químico, Professor Doutor, Instituto Federal de Educação, Ciência e Tecnologia do Ceará, Maracanaú, CE, Brasil.

\footnotetext{
* Contribuição técnica ao 69 Congresso Anual da ABM - Internacional e ao 14ํㅡㄹ ENEMET - Encontro Nacional de Estudantes de Engenharia Metalúrgica, de Materiais e de Minas, 21 a 25 de julho de 2014, São Paulo, SP, Brasil.
} 


\section{INTRODUÇÃO}

Os equipamentos de refino usados nas indústrias petroquímicas geram altos custos de manutenção, pois os mesmos apresentam desgaste devido à corrosão causada pelo elevado índice de acidez do petróleo extraído.

Ainda que o custo para recuperar equipamentos comprometidos pela corrosão provocada pela acidez do petróleo seja alto, o processamento de petróleo de alta acidez é economicamente atrativo. Uma possível solução para resolver este problema seria a procura por novos materiais para fabricar componentes que suportem esta adversidade e retardem o efeito da corrosão diminuindo os gastos com a manutenção de equipamentos, favorecendo assim a cadeia produtiva do setor de Petróleo e Gás Natural [1]. Como sugestão tem-se a utilização de ligas inoxidáveis ferríticas com altos percentuais de molibdênio, pois segundo Gunn [2], proporciona a característica de resistência à corrosão por pite e à corrosão galvânica para o aço em solução contendo cloreto. O molibdênio amplia o intervalo do potencial passivo e reduz a taxa de corrosão.

Portanto, a partir de demandas provenientes da indústria do petróleo foram desenvolvidas duas novas ligas Fe-Cr-Mo com um elevado teor de molibdênio $5 \%$ um percentual superior ao de ligas comerciais. Uma liga com $17 \%$ de cromo e a outra com $15 \%$ de cromo, denominadas, respectivamente, Liga 1 e Liga 2.

Por se tratar de ligas experimentais não dispomos de material de adição com composição semelhante para emprego na soldagem, sendo necessário emprego de material de adição dissimilar. Uma opção para a soldagem dessas ligas seria utilizar um eletrodo de superliga de níquel com alto teor de molibdênio para evitar a perda desse elemento durante a soldagem. Como os materiais de base e de solda são diferentes é possível que na interface solda-material de base haja a formação de uma pilha eletroquímica e, como são ligas novas não conhecemos o comportamento eletroquímico das mesmas.

Portanto, o objetivo deste trabalho foi analisar o comportamento corrosivo na solda dissimilar de duas novas ligas de aço inoxidável ferrítico com diferentes teores de cromo e um elevado teor de molibdênio, ambas com adição de superliga de níquel, verificando a influência da pilha.

\section{MATERIAL E MÉTODOS}

Para a realização dos experimentos foram utilizadas duas ligas experimentais de aço, cuja composição química é descrita na Tabela 1.

Tabela 1. Composição química (\% em massa) das ligas Fe-Cr-Mo [1]

\begin{tabular}{c|c|c|c|c|c}
\hline LIGA & C & Mn & Si & Cr & Mo \\
\hline 1 & 0,031 & 0,30 & 0,39 & 17,6 & 5,3 \\
\hline 2 & 0,031 & 0,30 & 0,38 & 15,6 & 5,3 \\
\hline
\end{tabular}

As ligas foram recebidas na condição forjada e laminadas a quente com temperatura inicial de laminação de $1000^{\circ} \mathrm{C}$. Depois foram retirados corpos de prova e realizados tratamentos térmicos de solubilização. Este tratamento consistiu no aquecimento dos corpos de prova, até uma temperatura onde exista apenas a matriz do material por um período de 15 minutos, seguido de um resfriamento rápido em água até atingir a temperatura ambiente. A solubilização foi realizada com o objetivo de eliminar os

\footnotetext{
* Contribuição técnica ao $69^{\circ}$ Congresso Anual da ABM - Internacional e ao 14ํㅡㄹ ENEMET - Encontro Nacional de Estudantes de Engenharia Metalúrgica, de Materiais e de Minas, 21 a 25 de julho de 2014, São Paulo, SP, Brasil.
} 


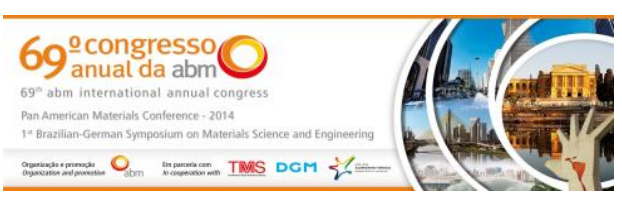

precipitados formados durante a laminação a quente, para então serem usinados os corpos de prova.

Após a solubilização as ligas foram submetidas a soldagem com adição de material através do processo MIG, que utiliza um arco elétrico como fonte de calor para promover a união entre um eletrodo consumível e a peça, onde um gás inerte é responsável pela proteção do arco e da região da solda. A Tabela 2 descreve 0 material de adição.

Tabela 2. Composição química do material de adição segundo o fabricante (\% em massa) [1]

\begin{tabular}{c|c|c|c|c|c|c|c|c|c|c|c}
\hline ELETRODO & $\mathrm{C}$ & $\mathrm{Si}$ & $\mathrm{Mn}$ & $\mathrm{P}_{\text {máx }}$ & $\mathrm{S}_{\text {máx }}$ & $\mathrm{Cr}$ & $\mathrm{Ni}$ & $\mathrm{Mo}$ & $\mathrm{Nb}$ & $\mathrm{Ti}$ & $\mathrm{Fe}$ \\
\hline $\begin{array}{c}\text { AWS } \\
\begin{array}{c}\text { ERNiCrMo-3 } \\
\text { (Inconel 625) }\end{array}\end{array}$ & $<0,03$ & 0,2 & 0,2 & 0,015 & 0,015 & 22,0 & $>60,0$ & 9,0 & 3,5 & $<0,20$ & $<1,0$ \\
\hline
\end{tabular}

A escolha do eletrodo foi feita com base nos requisitos de excelente qualidade da junta soldada, nos teores de molibdênio e boas propriedades mecânicas. Uma vez que este eletrodo tem um alto teor de molibdênio, espera-se evitar problemas com a perda de molibdênio do metal de base na realização das soldagens. A velocidade de alimentação do arame foi de $7,5 \mathrm{~m} / \mathrm{min}$, com tensão $(\mathrm{U})$ de $30 \mathrm{~V}$, vazão de gás $(\mathrm{Vg})$ de $20 \mathrm{l} / \mathrm{min}$ e distância de bico de contato peça (DBCP) de $20 \mathrm{~mm}$. Os parâmetros empregados nos cordões depositados na raiz das juntas soldadas são observados na Tabela 3 e as condições de soldagem utilizadas podem ser vistas na Tabela 4.

Tabela 3. Parâmetros de soldagem das juntas soldadas [1]

\begin{tabular}{c|c|c|c}
\hline CONDIÇÃO & $\mathrm{V}(\mathrm{cm} / \mathrm{min})$ & $\mathrm{I}(\mathrm{A})$ & $\mathrm{E}(\mathrm{KJ} / \mathrm{cm})$ \\
\hline $\mathrm{R}$ & 60 & 210 & 6,30 \\
\hline
\end{tabular}

Tabela 4. Condições de soldagem no processo MIG [1]

\begin{tabular}{c|c|c|c|c}
\hline CONDIÇÃO & Vs $(\mathrm{cm} / \mathrm{mim})$ & Is $(\mathrm{A})$ & Es $(\mathrm{KJ} / \mathrm{cm})$ & $\begin{array}{c}\text { Técnica de } \\
\text { Energia }\end{array}$ \\
\hline M1 & 38 & 210 & 9,9 & $\mathrm{~V}$ \\
\hline M5 & 25 & 280 & 20,2 & $\mathrm{I}$ \\
\hline
\end{tabular}

Foram confeccionados corpos de prova com chanfro meio $\mathrm{V}$ em forma de barras chatas com espessura de $12 \mathrm{~mm}$, largura de $70 \mathrm{~mm}$, comprimento de $250 \mathrm{~mm}$ de acordo com as normas AWS B2. 1: 2005 e ASME IX: 2004. A soldagem da junta foi realizada utilizando corrente contínua, eletrodo positivo (CCEP) e argônio como gás de proteção. A geometria da junta soldada pode ser vista na Figura 1.

\footnotetext{
* Contribuição técnica ao 69 Congresso Anual da ABM - Internacional e ao 14ํㅡㄹ ENEMET - Encontro Nacional de Estudantes de Engenharia Metalúrgica, de Materiais e de Minas, 21 a 25 de julho de 2014, São Paulo, SP, Brasil.
} 

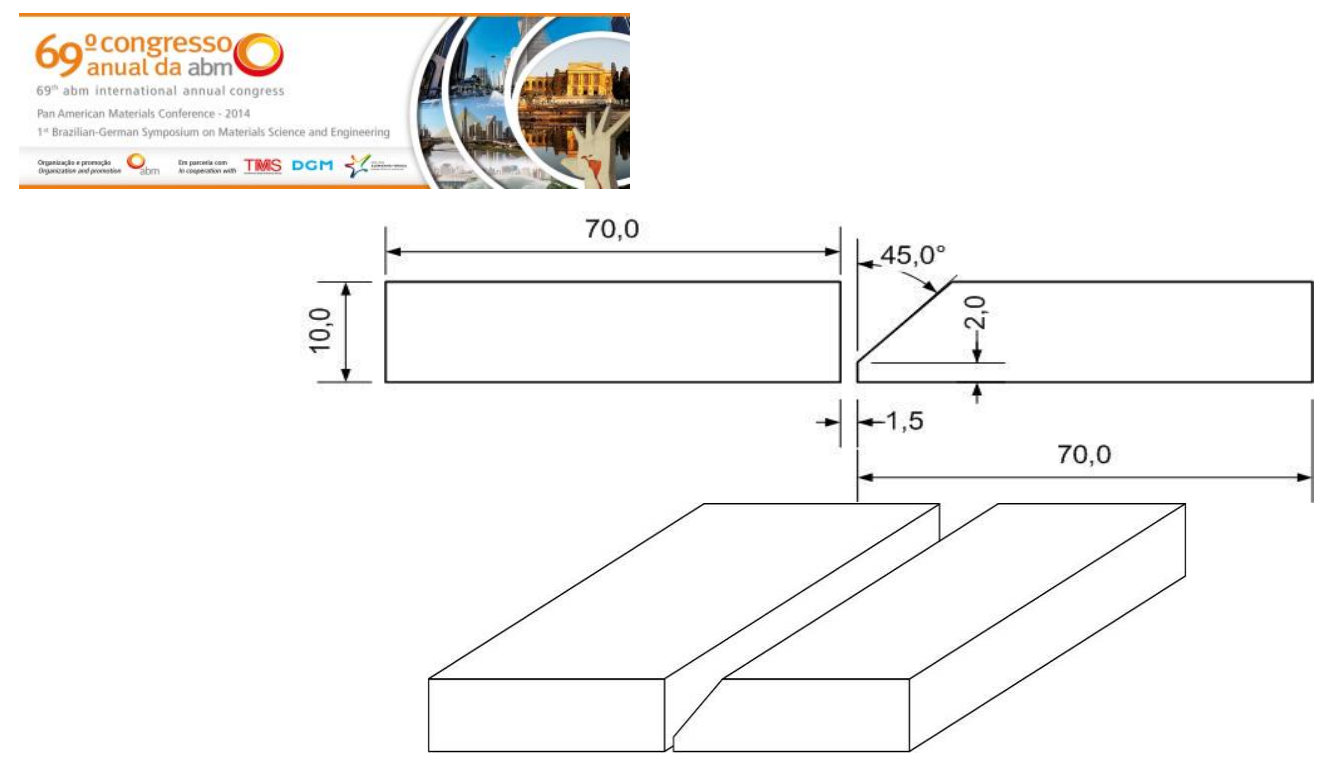

Figura 1. Geometria da junta soldada com adição de material [1]

Para a realização dos ensaios eletroquímicos na Liga 1 e na Liga 2, foi utilizado um potenciostato/galvanostato AUTOLAB modelo PGSTAT 30, a solução eletroquímica usada foi uma mistura de $0,5 \mathrm{~mol} / \mathrm{L}$ de $\mathrm{H}_{2} \mathrm{SO}_{4}+0,5 \mathrm{~mol} / \mathrm{L}$ de $\mathrm{NaCl}$, com um ph entre 0,3 e 0,5 .

Foram realizados ensaios de Polarização Potenciodinâmica Cíclica (PPC) utilizando o programa GPES para controle dos ensaios e aquisição de dados. A varredura potencial iniciou-se $200 \mathrm{mV}$ abaixo do potencial de corrosão (Ecorr) e se estendeu até que a corrente anódica alcançasse o valor de aproximadamente $1 \mathrm{~mA}$, no qual foi aplicada a polarização de reversão. A velocidade de varredura utilizada no experimento foi de $1,67 \mathrm{mV} . \mathrm{s}^{-1}$. Após a realização dos ensaios de polarização, foi feita uma nova caracterização microestrutural em amostras da Liga 1 e da Liga 2.

\section{RESULTADOS E DISCUSSÃO}

\subsection{Caracterização das Ligas Solubilizadas}

Foram retiradas amostras das Ligas 1 e 2 para a realização de análises metalográficas por meio de microscopia ótica (MO), microscopia eletrônica de varredura (MEV) e difração de elétrons retro-espalhados (EBSD), o que foi possível após a preparação das superfícies, com lixamento e ataque químico utilizando o reagente Vilella [3].

A Figura 2 apresenta micrografias da seção transversal da chapa correspondente as Ligas 1 e 2, após a realização do tratamento de solubilização.
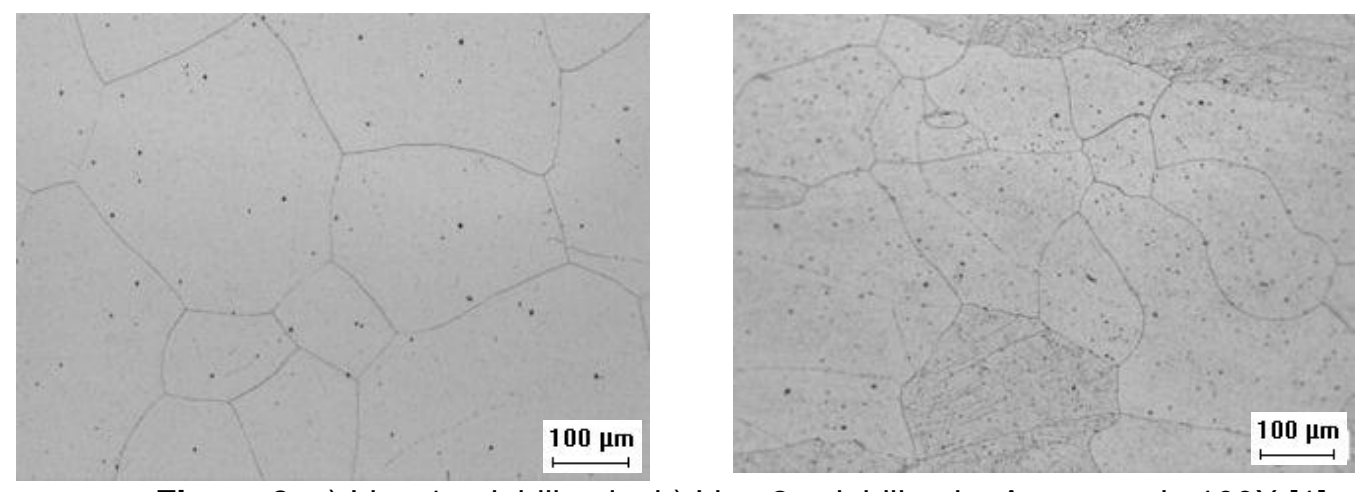

Figura 2. a) Liga 1 solubilizada. b) Liga 2 solubilizada. Aumento de 100X [1].

\footnotetext{
* Contribuição técnica ao $69^{\circ}$ Congresso Anual da ABM - Internacional e ao 14ํㅡㄹ ENEMET - Encontro Nacional de Estudantes de Engenharia Metalúrgica, de Materiais e de Minas, 21 a 25 de julho de 2014, São Paulo, SP, Brasil.
} 
Observa-se, na Figura 2, que tanto a Liga 1 quanto a Liga 2 apresentam uma microestrutura do tipo ferrítica com a presença de pequenos precipitados distribuídos ao longo dos grãos, possivelmente formados devido ao aumento do teor de molibdênio nas ligas [4].

$\mathrm{O}$ alto teor de molibdênio combinado com o cromo presente nas ligas e a ausência de elementos estabilizadores dos grãos resultam em ligas com grãos extremamente grandes [1]. O que é confirmado após a realização da medição do tamanho de grão das ligas, feita através do método ASTM e com um programa empregado na técnica de EBSD, a Liga $1 \mathrm{com}$ valor de 1,2 e a Liga 2 com valor de 1,6.

O aumento no tamanho dos grãos pode reduzir a cinética de decomposição da ferrita em fases deletérias o que pode ter favorecido aos precipitados observados na microestrutura das ligas estudadas [5].

Também foram obtidos resultados através do EBSD, para análise do possível surgimento de fases precipitadas na microestrutura das ligas, o que não foi verificado quando observados os resultados indicados na Tabela 5. O resultado da Tabela confirma que as ligas estudadas são do tipo ferrítica. A não identificação dos precipitados observados, menos de 1\% (Tabela 5), está associado a pequena quantidade que foram formados, impossibilitando a sua identificação através do EBSD.

Tabela 5. Identificação das fases, através do EBSD, das ligas solubilizadas [1]

\begin{tabular}{c|c|c}
\hline \multirow{2}{*}{ LIGA } & \multicolumn{2}{|c}{ PERCENTUAL DAS FASES } \\
\cline { 2 - 3 } & Ferro- $\alpha$ & Não Identificada \\
\hline 1 & 99,9 & 0,1 \\
\hline 2 & 99,6 & 0,4 \\
\hline
\end{tabular}

\subsection{Caracterização Microestrutural das Ligas Soldadas}

A partir dessa caracterização foram analisados os resultados referentes aos parâmetros M1 e M5. Os mesmos correspondem a menor e a maior Energia de soldagem (Es), respectivamente de acordo como está apresentado na Tabela 4. Portanto, amostras da Liga 1 e da Liga 2, após soldagem com adição de material também passaram por uma caracterização microestrutural por meio de microscopia ótica, o que foi possível após preparação da superfície com lixamento e ataque químico.

As Figuras 3 e 4 ilustram micrografias das Ligas 1 e 2 após o processo de soldagem, nos parâmetros M1 e M5. São representadas as regiões do metal de solda com a zona afetada pelo calor (MS-ZAC) e o metal de base com a zona afetada pelo calor (MB$\mathrm{ZAC})$.

* Contribuição técnica ao 69 Congresso Anual da ABM - Internacional e ao 14ํㅡㄹ ENEMET - Encontro Nacional de Estudantes de Engenharia Metalúrgica, de Materiais e de Minas, 21 a 25 de julho de 2014, São Paulo, SP, Brasil. 

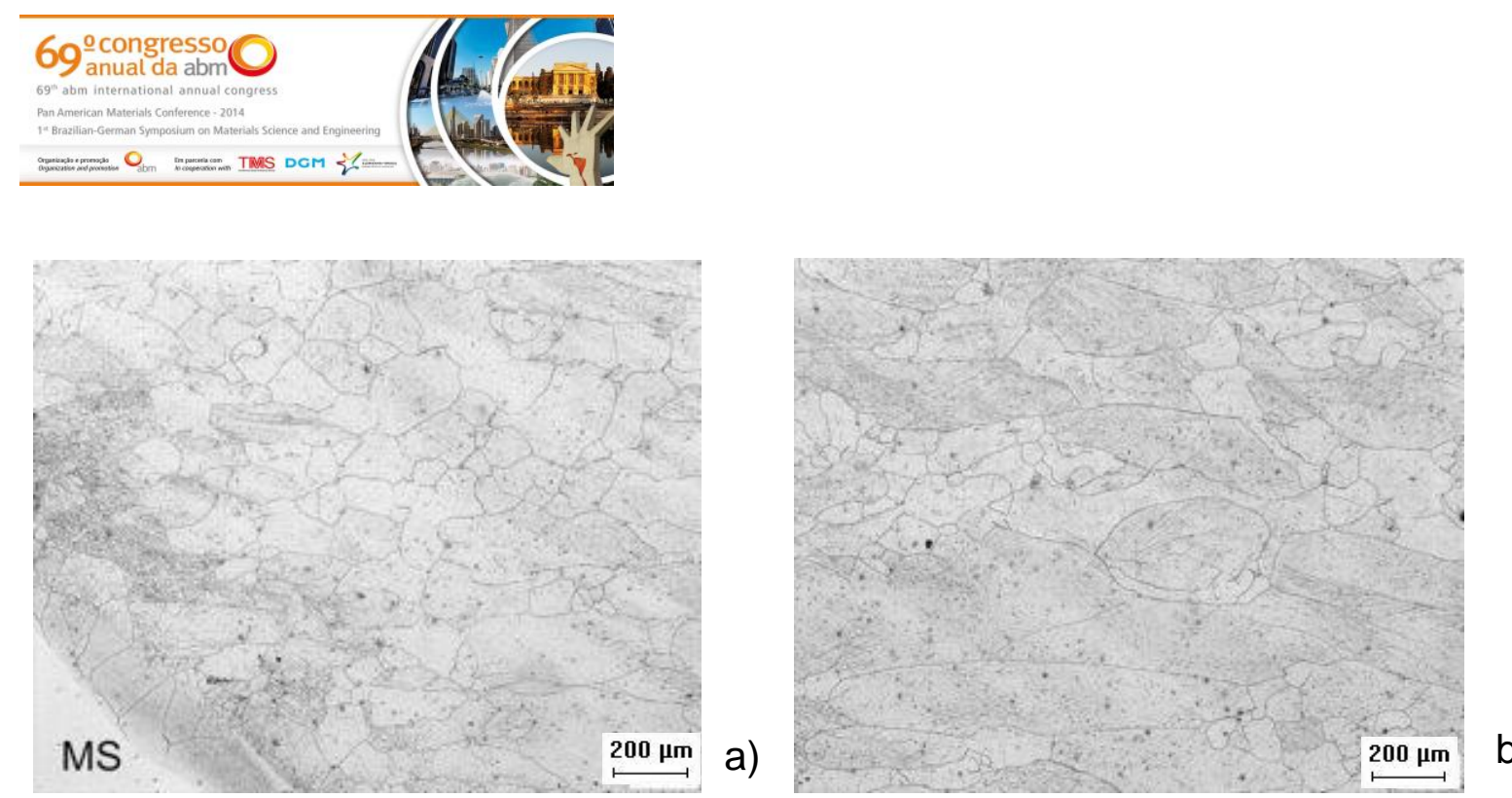

b)

Figura 3. Micrografia da solda com adição de material da Liga 1, parâmetro M1. Aumento de 50X. Ataque: Vilella. a) MS-ZAC. b) ZAC-MB [1].

Tanto na Liga 1 quanto na Liga 2 não foi possível identificar uma diferença significativa entre a ZAC e o MB. Independente da região é possível observar que os grãos apresentam diferentes tamanhos. A ausência de elementos estabilizadores do grão pode favorecer a este comportamento no tamanho do grão das ligas em estudo [6].
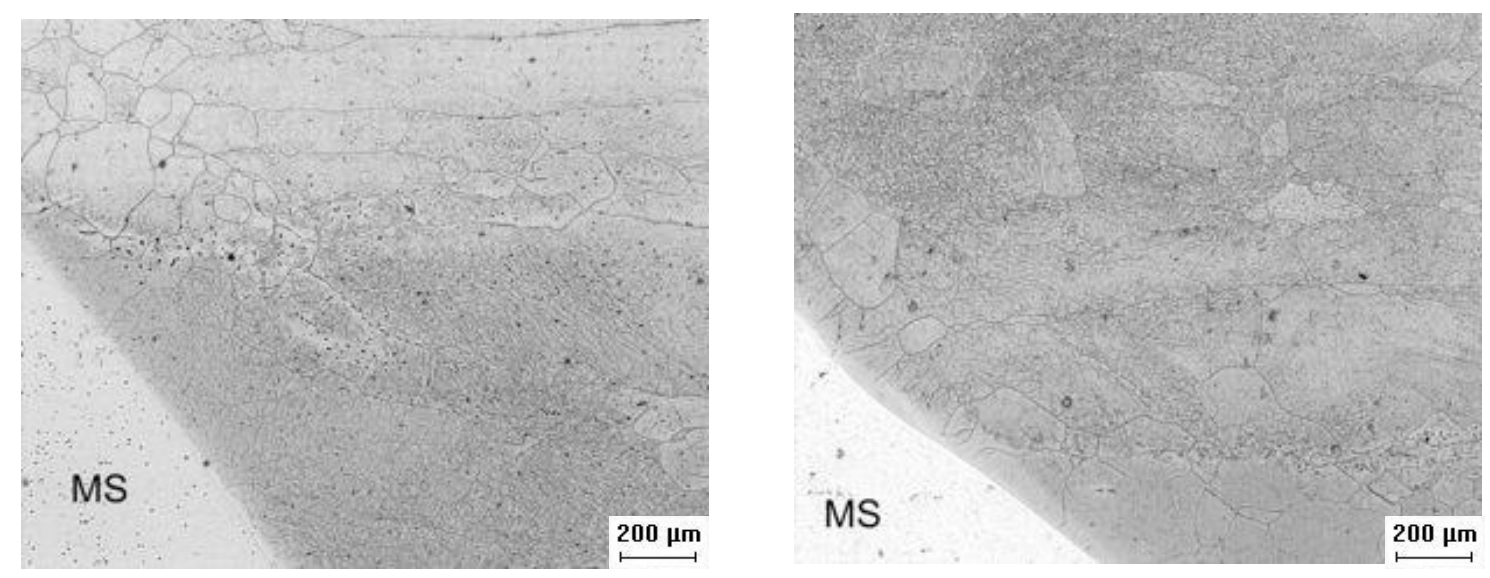

Figura 4. Micrografia da solda com adição de material, parâmetro M5. Aumento de 50X. Ataque: Vilella. Região MS-ZAC. a) Liga 1 b) Liga 2 [1].

O resultado para o parâmetro M1 foi semelhante ao obtido no parâmetro M5, também com uma microestrutura ferrítica de grãos grandes. A variação nos parâmetros de soldagem não resultou em mudanças significativas na microestrutura da Liga 1 e da Liga 2.

\subsection{Caracterização Microestrutural do Metal de Adição}

O metal de adição utilizado, o eletrodo AWS ERNiCrMo-3 (Inconel 625) teve sua microestrutura revelada após ataque químico com ácido crômico [3]. Na Figura 5 são apresentadas as micrografias da Liga 1 e da Liga 2 para o parâmetro M1 e na Figura 6 as micrografias para o parâmetro M5.

\footnotetext{
* Contribuição técnica ao $69^{\circ}$ Congresso Anual da ABM - Internacional e ao 14ํㅡㄹ ENEMET - Encontro Nacional de Estudantes de Engenharia Metalúrgica, de Materiais e de Minas, 21 a 25 de julho de 2014, São Paulo, SP, Brasil.
} 
a)

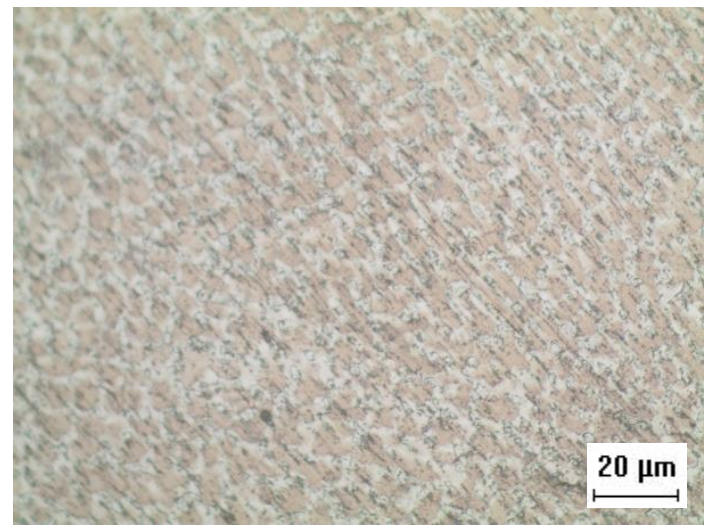

b)

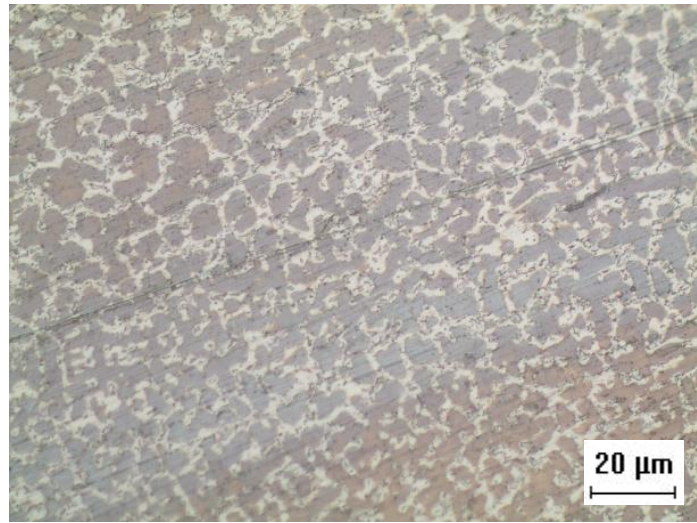

Fonte: Laboratório de Materiais do Instituto Federal - Campus de Maracanaú.

Figura 5. Micrografias do metal de adição. Aumento 500X. Ataque: Ácido crômico. Parâmetro M1. a) Liga 1 b) Liga 2.

a)

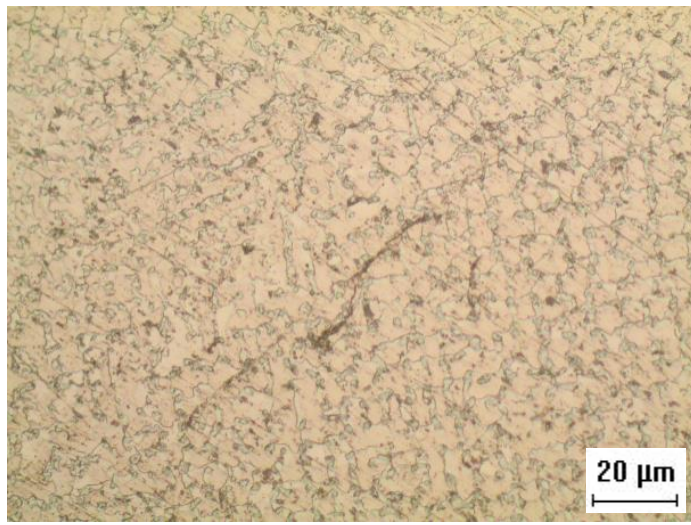

b)

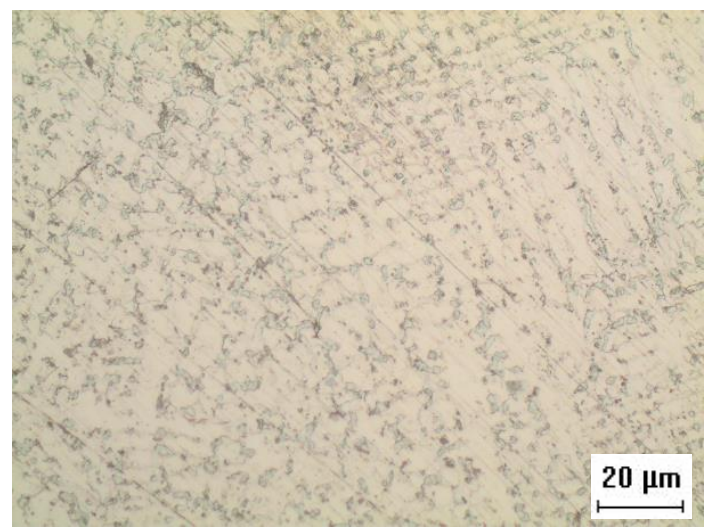

Fonte: Laboratório de Materiais do Instituto Federal - Campus de Maracanaú.

Figura 6. Micrografias do metal de adição. Aumento 500X. Ataque: Ácido crômico. Parâmetro M5. a) Liga 1 b) Liga 2.

Nas micrografias de ambos os parâmetros, tanto da Liga 1 quanto da Liga 2, é possível observar uma microestrutura dendrítica, pois segundo Boeira diferentes configurações microestruturais resultantes de processos de solidificação são formadas na movimentação da interface sólido/líquido. Se a liga metálica se solidifica em condições de equilíbrio termodinâmico, esta interface estabiliza-se na forma planar porém, a ocorrência de instabilidades ocasiona uma degeneração e conforme o caso, interferência em pontos localizados na frente sólida. Estas instabilidades são induzidas pela ocorrência de um superesfriamento e dependem da relação entre o gradiente de temperatura à frente desta interface e da velocidade de deslocamento da mesma. Desta forma a frente de solidificação pode desdobrar-se de planar para celular ou dendrítica. $O$ crescimento celular é definido pela ocorrência de grãos alongados com baixas velocidades de crescimento e perpendiculares ao fluxo de calor [7].

* Contribuição técnica ao 69을 Congresso Anual da ABM - Internacional e ao 14ํㅡㄹ ENEMET - Encontro Nacional de Estudantes de Engenharia Metalúrgica, de Materiais e de Minas, 21 a 25 de julho de 2014, São Paulo, SP, Brasil. 


\subsection{Análise dos Ensaios Eletroquímicos}

Inicialmente, foram realizados ensaios de Polarização Potenciodinâmica Cíclica (PPC) em amostras do metal de adição (Inconel 625) e em amostras do metal de base (MB) das Ligas 1 e 2 antes da soldagem, para analisar o comportamento corrosivo dos materiais que constituem os corpos de prova das ligas em estudo. Os gráficos são apresentados na Figura 7 e os valores obtidos referentes ao potencial de corrosão (Ecorr) e as densidades de corrente de passivação, são apresentados na Tabela 6.

a)
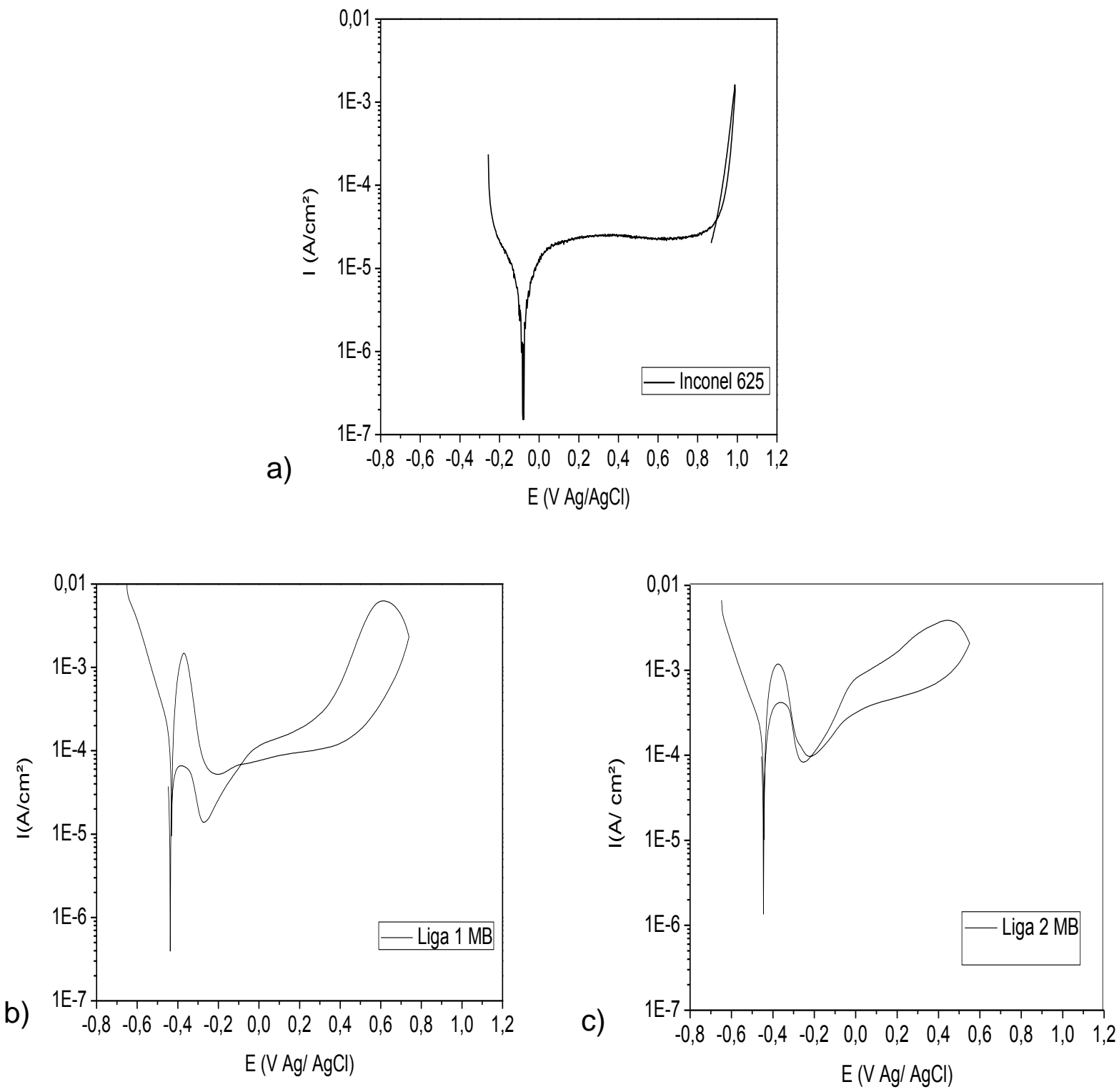

Fonte: Laboratório de Corrosão da Universidade Federal do Ceará.

Figura 7. Curvas de Polarização. a) Inconel 625. b) Liga 1 solubilizada. c) Liga 2 solubilizada.

\footnotetext{
* Contribuição técnica ao $69^{\circ}$ Congresso Anual da ABM - Internacional e ao 14ํㅡㄹ ENEMET - Encontro Nacional de Estudantes de Engenharia Metalúrgica, de Materiais e de Minas, 21 a 25 de julho de 2014, São Paulo, SP, Brasil.
} 


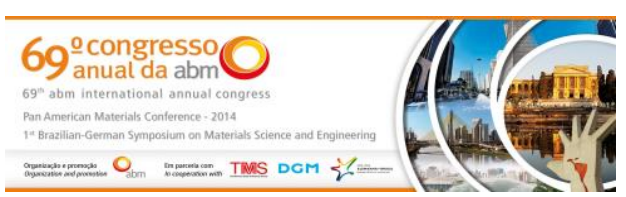

Tabela 6. Potenciais de corrosão e densidades de corrente dos materiais da solda.

\begin{tabular}{|c|c|c|}
\hline MATERIAL & $\begin{array}{c}\text { POTENCIAL DE } \\
\text { CORROSÃO }(\mathrm{V})\end{array}$ & $\begin{array}{c}\text { DENSIDADE DE CORRENTE } \\
\text { DE PASSIVAÇÃO }\left(\mathrm{A} / \mathrm{cm}^{2}\right)\end{array}$ \\
\hline Inconel & $-0,056$ & ${ }^{\star} 2,5 \times 10^{-5}$ \\
\hline Liga 1 solubilizada & $-0,451$ & $\mathrm{De}^{*} 5,5 \times 10^{-5} \mathrm{a} 1,5 \times 10^{-4}$ \\
\hline Liga 2 solubilizada & $-0,448$ & $\mathrm{De}^{*} 1,2 \times 10^{-4} \mathrm{a} 4,3 \times 10^{-4}$ \\
\hline
\end{tabular}

${ }^{\star}$ Aproximadamente.

Ao analisarmos as curvas da Figura 7, é possível verificar que o Inconel 625 apresenta uma região de passivação maior do que no $\mathrm{MB}$ da Liga 1 e da Liga 2, variando aproximadamente do potencial de $0,05 \mathrm{~V}$ até $0,9 \mathrm{~V}$ associado a densidades de correntes de passivação menores por volta de $2,5 \times 10^{-5} \mathrm{~A} / \mathrm{cm}^{2}$. Já o metal base das ligas apresenta uma faixa de potencial de aproximadamente $-0,2 \mathrm{~V}$ até $0,5 \mathrm{~V}$ na Liga 1 e até $0,4 \mathrm{~V}$ na Liga 2. Também foi possível observar que ao ser aplicada a polarização de reversão quando a corrente atingiu $1 \mathrm{~mA}$, apenas nas curvas do MB exibe-se um aumento de corrente significativo. Este resultado, por sua vez, sugere que ocorre um aumento da área ativa do eletrodo. Este aumento pode estar associado à formação de corrosão localizada, como alvéolos e pites.

Desta forma, de acordo com a discussão apresentada anteriormente, verificou-se claramente que no meio corrosivo empregado o metal de adição (Inconel 625) utilizado na soldagem apresenta uma maior resistência à corrosão do que o metal de base das ligas em estudo, pois de acordo com os gráficos da Figura 7 o Inconel é mais catódico, com região de passivação mais estável rompendo em potenciais mais elevados.

Para fins de comparação, foram realizadas curvas de PPC na interface formada pelas regiões da ZAC e o metal de adição.

Nas Tabelas 7 e 8 verifica-se a identificação das amostras tanto da Liga 1 quanto da Liga 2, pois foram realizados ensaios de polarização em triplicatas de cada parâmetro, para ambas as ligas em estudo.

Tabela 7. Identificação das amostras da Liga 1.

\begin{tabular}{|c|c|c|c|}
\hline \multirow{4}{*}{ LIGA 1 } & Parâmetro & Amostra & Descrição \\
\cline { 2 - 4 } & \multirow{3}{*}{ M1 } & 1 & L1-M1.1 \\
\cline { 2 - 3 } & & 2 & L1-M1.2 \\
\cline { 2 - 4 } & \multirow{3}{*}{ M5 } & 3 & L1-M1.3 \\
\cline { 2 - 4 } & & 1 & L1-M5.1 \\
\cline { 2 - 3 } & & 2 & L1-M5.2 \\
\cline { 2 - 3 } & 3 & L1-M5.3 \\
\hline
\end{tabular}

Tabela 8. Identificação das amostras da Liga 2.

\begin{tabular}{|c|c|c|c|}
\hline \multirow{4}{*}{ LIGA 2 } & Parâmetro & Amostra & Descrição \\
\cline { 2 - 4 } & \multirow{3}{*}{ M1 } & 1 & L2 - M1.1 \\
\cline { 2 - 3 } & & 2 & L2 - M1.2 \\
\cline { 2 - 4 } & \multirow{3}{*}{ M5 } & 3 & L2 - M1.3 \\
\cline { 2 - 4 } & & 1 & L2 - M5.1 \\
\cline { 2 - 3 } & & 2 & L2 - M5.2 \\
\cline { 2 - 3 } & 3 & L2 - M5.3 \\
\hline
\end{tabular}

* Contribuição técnica ao $69^{\circ}$ Congresso Anual da ABM - Internacional e ao 14ํㅡㄹ ENEMET - Encontro Nacional de Estudantes de Engenharia Metalúrgica, de Materiais e de Minas, 21 a 25 de julho de 2014, São Paulo, SP, Brasil. 
As Figuras 8 e 9 apresentam os gráficos obtidos após os ensaios de polarização realizados na interface da solda para as Ligas 1 e 2. Tais resultados, como já esperado, não apresentaram reprodutibilidade, visto que nesta interface ocorre a formação de uma pilha galvânica.
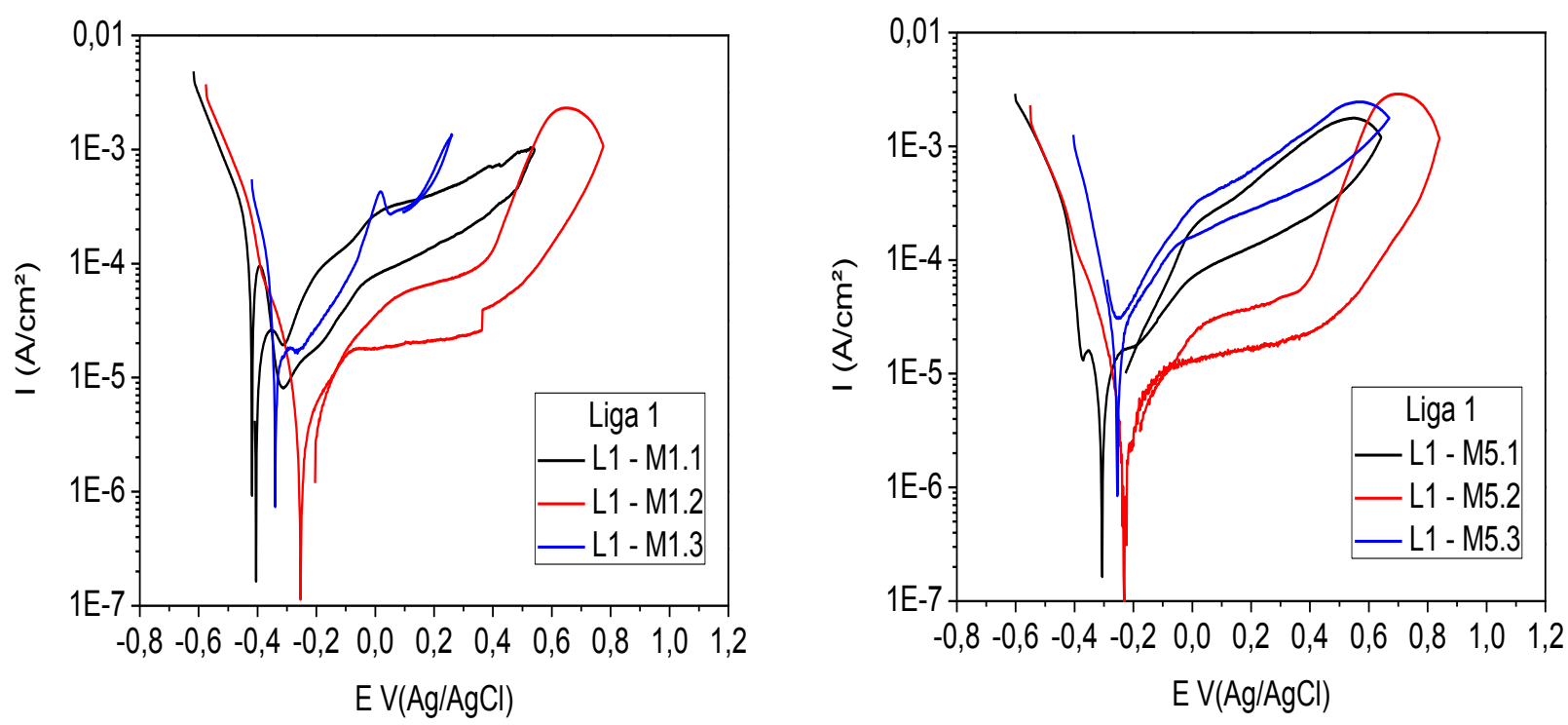

Fonte: Laboratório de Corrosão da Universidade Federal do Ceará.

Figura 8. Curvas de Polarização da Liga 1. a) Parâmetro M1. b) Parâmetro M5.
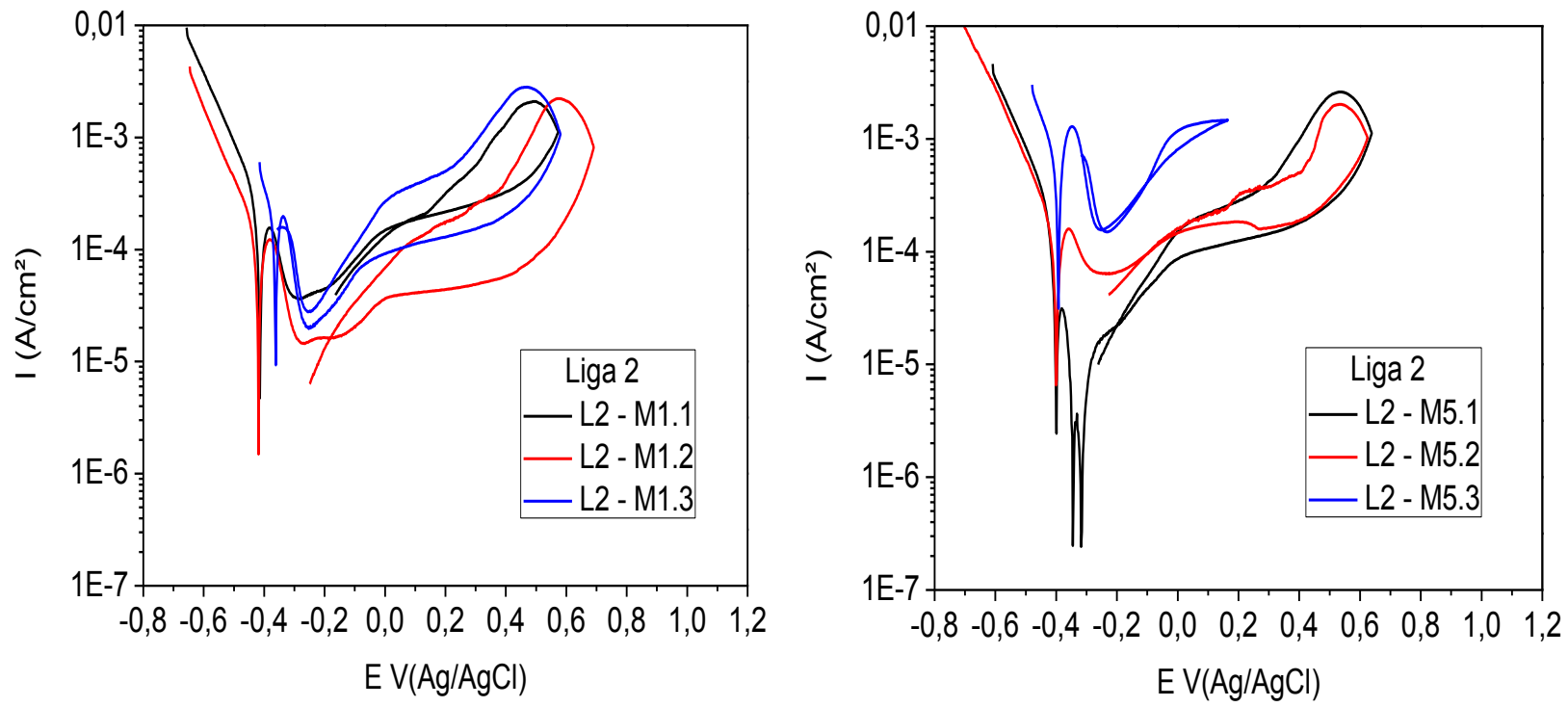

Fonte: Laboratório de Corrosão da Universidade Federal do Ceará.

Figura 9. Curvas de Polarização da Liga 2. a) Parâmetro M1. b) Parâmetro M5.

As Figuras 10 e 11 representam as micrografias obtidas após os ensaios de polarização em todas as imagens se observa uma linha vermelha que foi inserida para facilitar a visualização das regiões da solda (Inconel e ZAC).

* Contribuição técnica ao $69^{\circ}$ Congresso Anual da ABM - Internacional e ao 14ํㅡㄹ ENEMET - Encontro Nacional de Estudantes de Engenharia Metalúrgica, de Materiais e de Minas, 21 a 25 de julho de 2014, São Paulo, SP, Brasil. 


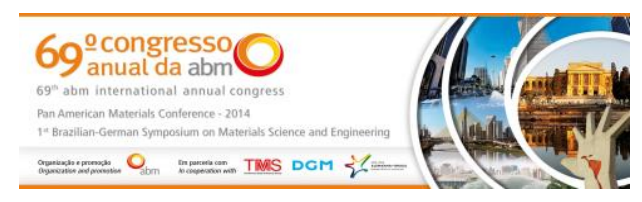

a)

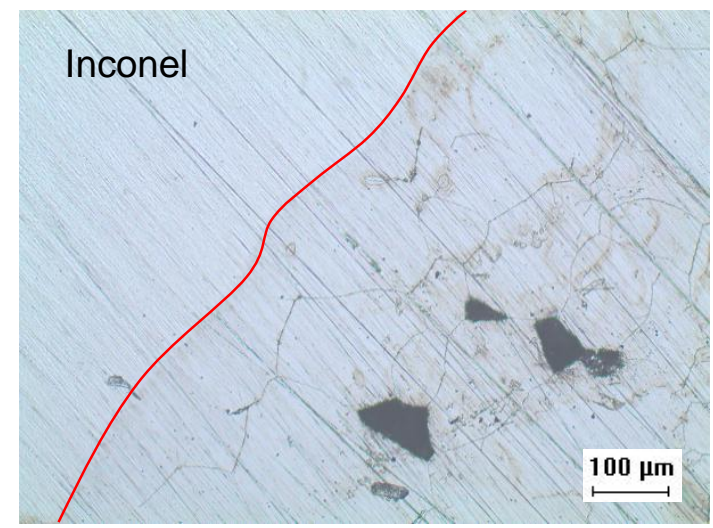

b)

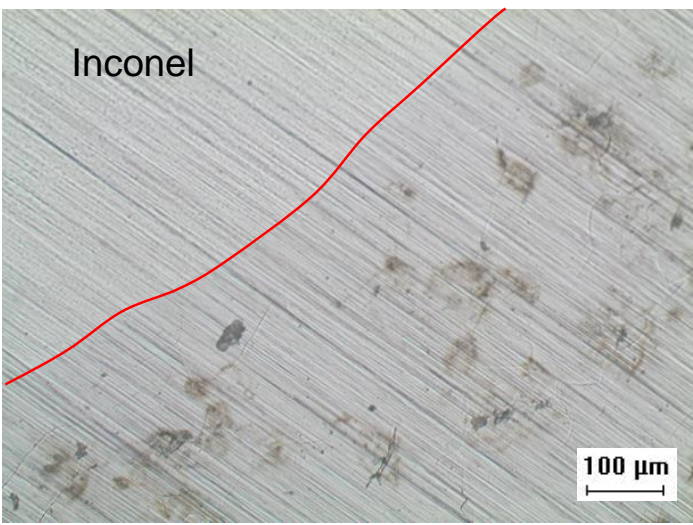

Fonte: Laboratório de Materiais do Instituto Federal - Campus de Maracanaú.

Figura 10. Micrografias após ensaio de polarização. Aumento de 100X. Parâmetro M1. a) Liga 1 b) Liga 2.

a)

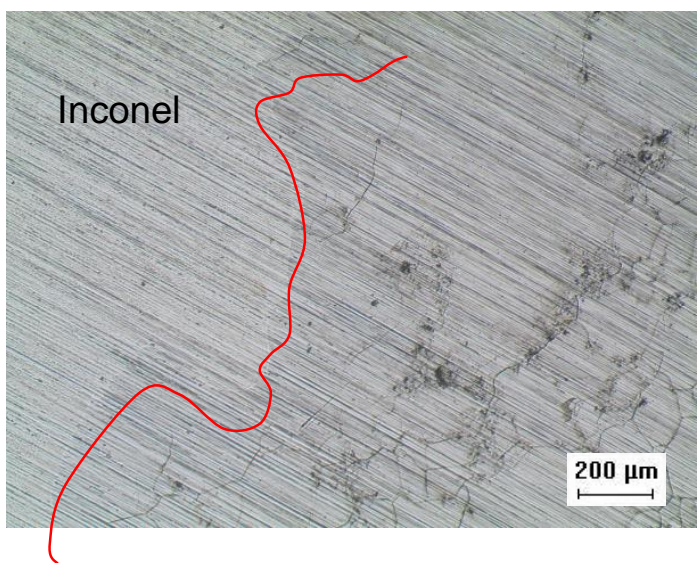

b)

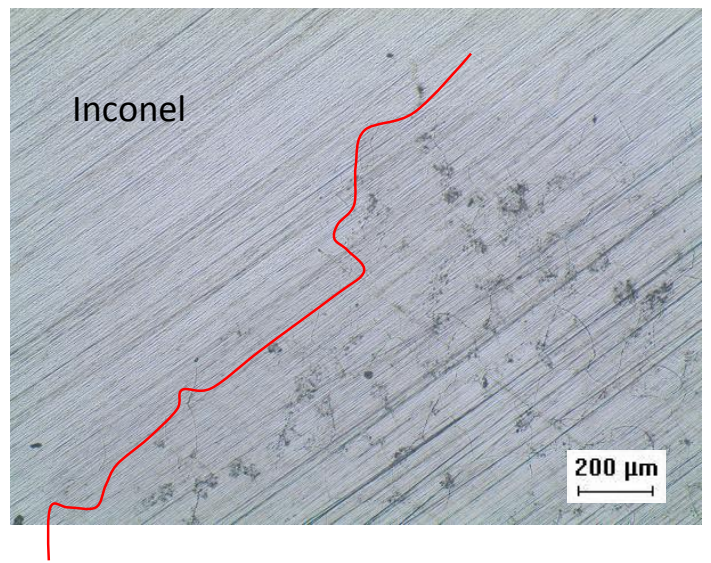

Fonte: Laboratório de Materiais do Instituto Federal - Campus de Maracanaú.

Figura 11. Micrografias após ensaio de polarização. Aumento de 50X. Parâmetro M5. a) Liga 1 b) Liga 2.

Apesar de todas as amostras visualmente apresentaram um aspecto corroído na região da ZAC, nas micrografias das interfaces verifica-se apenas na opção a da Figura 10 indícios de corrosão localizada na região da ZAC, pela presença de pontos escuros com diferentes dimensões.

A pilha galvânica presente na solda das Ligas 1 e 2 gera a necessidade de estudos mais aprofundados com relação a resistência a corrosão, pois a condição que elas apresentam, caracterizam um estudo novo sobre materiais com soldas dissimilares e que são submetidos a meios bastante agressivos.

\section{CONCLUSÕES}

A Liga 1 e a Liga 2 apresentaram uma microestrutura ferrítica, tanto na condição solubilizada quanto na soldada observando o metal de base (MB).

A caracterização microestrutural do metal de adição (Inconel 625) indicou a formação de uma microestrutura dendrítica em ambos os parâmetros estudados (M1 e M5).

\footnotetext{
* Contribuição técnica ao $69^{\circ}$ Congresso Anual da ABM - Internacional e ao 14ํㅡㄹ ENEMET - Encontro Nacional de Estudantes de Engenharia Metalúrgica, de Materiais e de Minas, 21 a 25 de julho de 2014, São Paulo, SP, Brasil.
} 


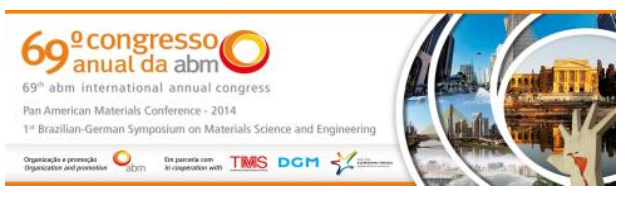

A curva de polarização obtida a partir do metal de adição indicou que o mesmo apresenta elevada resistência à corrosão por apresentar uma região de passivação mais estável que rompe em potenciais elevados.

Os ensaios eletroquímicos realizados na interface da solda das ligas, não resultaram em curvas de polarização homogêneas nas amostras de cada parâmetro empregado. A análise das superfícies das ligas submetidas aos ensaios, ressaltaram a necessidade do uso de novas técnicas de análise.

Como sugestão tem-se a realização de novos ensaios eletroquímicos como Espectroscopia de Impedância Eletroquímica e também o monitoramento do potencial das ligas enquanto imersas em solução por um tempo a ser determinado.

\section{Agradecimentos}

Os autores agradecem aos Laboratórios de Materiais (LMAT) e de Inspeção e Análise de Falhas (LIAF) do IFCE Campus de Maracanaú e ao Laboratório de Corrosão da UFC pela realização dos experimentos, ao GIAF (Grupo de Inspeção e Análise de Falhas) também do IFCE pelo suporte técnico, além do CNPq e a PETROBRAS pelo incentivo financeiro.

\section{REFERÊNCIAS}

1 Guimarães RF. Efeito do teor de molibdênio na microestrutura de juntas soldadas em ligas Fe-Cr-Mo [Tese de Doutorado]. UFC; 2011.

2 Gunn R. Duplex Stainless Steels (Microstructure, properties and applications, 1997, Abington Publishing.

3 Voort GFV, James H, Mills MK, Davis JR, Destefani JD, Dieterich DA, et al.. (Eds). ASM Handbook: Metallography and Microstructures, USA: ASM International. 1992; 9: 284, 587.

4 Costa E Silva AL, Mei PR. Aços e ligas Especiais. 6ª.., Edgar Blucher, São Paulo, 2006.

5 Pardal, J.M., TAVARES, S. S. M., CINDA FONSECA, M., de SOUZA, J. A., CÔRTE , R. R. A., ABREU, H. F. G. "Influence of the grain size on deleterius phase precipitation in superduplex stainless steel UNS S322750. Materials Characterization, 2008.

6 Villafuerte JC, Kerr HW. "Phase transformations in stainless steel weld metal and heataffected zones". Key Engineering Materials, v.69\&70, p.129-148, 1992.

7 Boeira AP. Análise numérica e experimental da solidificação das ligas AI 3,5\% Cu E AA356 [Dissertação Mestrado]. Porto Alegre: Universidade Feral do Rio Grande do Sul; 2002.

\section{BIBLIOGRAFIA}

1 Guimarães RF. Avaliação comparativa dos linings dos aços AISI 444 e AISI e AISI 316L para aplicação e torres de destilação de petróleo: 2005. 109p. Dissertação (Mestrado) Universidade Federal do Ceará, Fortaleza.

2 Garcia A. Solidificação: Fundamentos e Aplicações, 1a edição, Campinas, Brasil, Ed. Unicamp, 2001.

3 Silva SC. Mecanismos de trincamento de aço de microestrutura austeno-ferrítica em meio corrosivo. 2011. 115p. Projeto de Graduação - Universidade Federal do Rio de Janeiro.

4 Moraes JTL. Soluções inovadoras: alternativa ao aço. Notícias da Construção, 2013; 10(123): 44-45.

5 Panossian ZP, Vargas C; Brunelli RA; Almeida NL. Estudo da corrosão em frestas de ligas de alta resistência à corrosão por meio de ensaios eletroquímicos. In: Conferência Sobre Tecnologia De Equipamentos, 12, 2013, Porto de Galinhas. Anais... 11p.

\footnotetext{
* Contribuição técnica ao 69을 Congresso Anual da ABM - Internacional e ao 14ํㅡㄹ ENEMET - Encontro Nacional de Estudantes de Engenharia Metalúrgica, de Materiais e de Minas, 21 a 25 de julho de 2014, São Paulo, SP, Brasil.
} 
6 Araujo A, Mesquita TJ, Chauveau E, Mantel M; Panossian Z, Santos CAL, Nogueira RP. Carbonated and chloride contaminated concrete strucutre : the role of molybdenum in corrosion of stainless steel reinforcement. Corrosão e Protecção de Materiais, 2013; 32(2): 5-8.

7 Cardoso JL, Almeida LF, Moreira AR; Almeida NL; Guedes FM; Vaz GL, et al.. Avaliação de inibidores de corrosão e da influência do tempo de pré-corrosão na prevenção de corrosão preferencial em juntas soldadas aplicadas a sistemas de produção de gás. In: Congresso Brasileiro De Corrosão, INTERCORR 2012, 32, 2012, Salvador. Anais... Salvador: ABRACO, 2012. 15p.

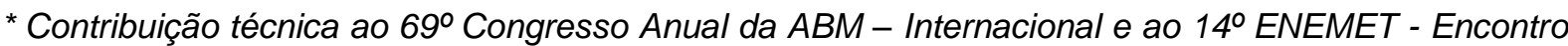
Nacional de Estudantes de Engenharia Metalúrgica, de Materiais e de Minas, 21 a 25 de julho de 2014, São Paulo, SP, Brasil. 